\title{
BMJ Open Dose-response relationship between urinary PAH metabolites and blood viscosity among coke oven workers: a cross-sectional study
}

Min Wu, Chengjuan Liu, Huimin Wang, Jisheng Nie (D) , Jin Yang

To cite: Wu M, Liu C, Wang $\mathrm{H}$, et al. Dose-response relationship between urinary PAH metabolites and blood viscosity among coke oven workers: a crosssectional study. BMJ Open 2021;11:e046682. doi:10.1136/ bmjopen-2020-046682

- Prepublication history and additional supplemental material for this paper are available online. To view these files, please visit the journal online (http://dx.doi.org/10.1136/ bmjopen-2020-046682).

Received 09 November 2020 Accepted 06 September 2021

D) Check for updates

(C) Author(s) (or their employer(s)) 2021. Re-use permitted under CC BY-NC. No commercial re-use. See rights and permissions. Published by BMJ.

School of Public Health, Shanxi Medical University, Taiyuan, Shanxi, China

Correspondence to

Dr Jisheng Nie;

niejisheng@sxmu.edu.cn

\section{ABSTRACT}

Objectives Polycyclic aromatic hydrocarbons (PAHs) have been proven to be a risk factor for cardiovascular disease in coke oven workers, and increased plasma viscosity is a signal for higher risk of catching up cardiovascular disease. We want to explore whether the plasma viscosity is affected by the concentration of PAHs.

Design Our study is a cross-sectional dose-response study.

Setting Participants in this study came from a coke plant in Taiyuan, Shanxi.

Participants We used data of 693 coke oven workers in Taiyuan.

Primary and secondary outcome measures We assumed that plasma viscosity would increase as the concentration of PAHs metabolites in urine increases. We found that 2-hydroxyfluorene (OHFLU2) and plasma viscosity have a stable linear relationship in different statistical methods.

Results We found that plasma viscosity increased by 1.14 (mPa.s,30/s) for each $\mathrm{ng} / \mathrm{mL}$ of 2-OHFLU urinary (correlation coefficient range: $0.54-1.74, p<0.05$ ). Conclusions The results of this study could provide evidence for coke oven workers to prevent cardiovascular disease by checking whether plasma viscosity is elevated.

\section{INTRODUCTION}

Polycyclic aromatic hydrocarbons (PAHs) are air pollutants commonly present in the environment, mainly originating from the incomplete combustion of organic materials such as coal and petroleum products. ${ }^{12} \mathrm{PAHs}$ can increase the risk of cardiovascular disease (CVD), as confirmed by many studies. A study by $\mathrm{Xu}$ et al found that in the USA, urinary metabolites of PAHs were associated with self-reported CVD. ${ }^{3}$ Another study in China suggested that some urinary monohydroxy PAH metabolites (OH-PAHs) have a relationship with arteriosclerotic CVD (ASCVD) risk. ${ }^{4}$ PAHs' association with arrhythmia has been confirmed in mammalian (including human) studies worldwide.$^{56}$ PAHs' association with CVD has been extensively investigated, but
Strengths and limitations of this study

- This was a cross-sectional study and cannot prove causal relationship.

- We use the high-performance liquid chromatography with tandem mass spectrometry to measure 11 urinary monohydroxy polycyclic aromatic hydrocarbons in worker urine samples.

- The data source used in this study is 693 healthy coking plant workers.

its relationship to plasma viscosity is not yet known.

Plasma viscosity measurement is an important test item for haemorheology testing. High plasma viscosity is usually used as a signal of coronary heart disease and stroke. Compared with healthy subjects, plasma viscosity is higher in patients with ischaemic heart disease, ${ }^{7}$ and the higher the plasma viscosity is, the worse the severity of coronary heart disease tends to be. ${ }^{8}$ Plasma viscosity also may play an important part in atherosclerosis, thrombosis and ischaemia. ${ }^{9}$ There are many studies showing that higher plasma viscosity is associated with higher risk of CVD (ie, coronary heart disease and stroke) and mortality in the general population. ${ }^{1011}$

The range of normal plasma viscosity is narrow and independent of age and gender. It is not influenced by haematocrit (anaemia, polycytemia), red blood cell aggregation, haemoglobinopathias or time to analysis. Changes in plasma viscosity indicate decreased cardiovascular function. Plasma viscosity could be used as a biomarker for CVD. Serum biomarkers of CVD-like C reactive protein (CRP), fibrinogen and homocysteine have been well known and used as biomarkers to prevent CVD in human populations. But these serum biomarkers are not found to have an association with PAHs. ${ }^{12}$ Our research focuses on whether there is a 
dose-effect relationship between plasma viscosity and PAHs. The results of our study may provide a new marker for coke oven workers to prevent CVD.

\section{METHOD}

\section{Study population}

All subjects of this study were recruited in a coking plant located in Shanxi, China. These workers have worked for the coking plant for more than 1 year. People were excluded who had a history of encephalopathy, liver or kidney diseases or autoimmune diseases. A total of 776 coke oven workers were investigated in this study, but 76 workers were excluded from this study because of lacking urine samples. Nine workers were excluded due to a lack of data of plasma viscosity. A total of 691 participants were included in the final analysis.All study participants from the study prior provided informed consent. The informed consent form is found in online supplemental materials.

Our research group designed a questionnaire, and this questionnaire has been practiced before the final investigation, which is used to obtain the subjects' sociodemographic characteristics during these workers' working period, for example, the age, sex, nationality, occupational history, length of service, smoking and drinking habits, frequency of eating fried and coal-baked foods, histories of personal and family diseases, symptoms, medication used, etc. We defined smoking as currently smoking no less than one cigarette per day over the last 6 months, and drinking as currently drinking wine, beer or spirits no less than three times a week for the last 6 months. Of 691 exposed workers included in the study completed the questionnaire.

\section{Biological sample collection and analysis}

We used a urine container to collect urine sample from all participants who took part in the occupational examination. The urinary samples were immediately transported to the laboratory, and urinary gravity was measured initially. After that, the urinary samples were repackaged into $5 \mathrm{~mL}$ cryogenic vials, and then stored at $-80^{\circ} \mathrm{C}$ until chemical analysis. Urine samples were measured for a suite of PAH metabolites: 2-hydroxynaphthalene (2-OH NAP), 1-OH NAP, 3-hydroxyfluorene (3-OH FLU), 2-OH FLU, 9-hydroxyphenanthrene (9-OH PHE), 2-OH PHE, 1-OH PHE, 1-hydroxypyrene (1-OH PYR), 3-hydroxychrysene (3-OH CHR), 6-OH CHR and 9-hydroxybenzo[a]pyrene (9-OH Bap), by high-performance liquid chromatography with tandem mass spectrometry (Shimadzu, Kyoto, Japan). ${ }^{13}$ High-performance liquid chromatography's separation conditions and mass spectrometry's detection programme were previously described in our study. ${ }^{14}$

\section{Blood and biochemical indicators}

Blood samples were collected from each participant according to the standard methods and used for routine blood examination (including white blood cell, red blood cell, absolute platelet count, absolute lymphocyte count, absolute neutrophil count, monocyte count, mean corpuscular volume, mean corpuscular haemoglobin $(\mathrm{MCH})$, MCH concentration) with a fully automated haematology analyser (XS-1000i, Sysmex Corporation, Kobe, Japan) and blood biochemical indicators (including alanine aminotransferase, aspartate aminotransferase, blood glucose, TCHO, TG, HDL, LDL) with a fully automated biochemical analyser (CS-400B, Dirui Industrial, Changchun, China). The measurement data of plasma viscosity in this study were provided by the cooperative hospital. The method used was lamina rotation (LBY-N6K automatic hemorheometer). This is the main method for whole blood viscosity detection. High, medium and low shear rate conditions are set for the determination, and the apparent viscosity of whole blood is measured at different shear rate.

\section{Statistical analysis}

We used SPSS V.22.0 (SPSS Chicago, Illinois) and SAS V.9.4 (SAS Institute, Cary, North Carolina) to analyse the data we collected. We used numbers and frequency (\%) to represent categorical variables and mean \pm SD for continuous variables. The detection rate of 3-OH CHR and 6-OH CHR in urine was less than 50\%; therefore, we did not include the two urinary PAH metabolites in the final analysis. We used one-half the LOD to assign the concentration of PAH metabolites below the LOD for other PAH metabolites. We used the generalised linear models and restrict cubic spline models to explore the dose-response association between PAH metabolites and plasma viscosity after adjusting for potential confounders. Covariates were selected including age, sex, smoking, drinking, BMI, education, income, fruit, grease, TG, TC, HDL, LDL, OH-PAHs. A bilateral $p$ value less than 0.05 was considered statistically significant.

\section{Patient and public involvement statement}

In this study, we collected data on coking plant workers. Coke plant workers who participated in the 2018 baseline survey may be selected as participants in this follow-up study. Workers who completely voluntarily fill in the informed consent form and provide blood and urine samples were involved in this study. Patients were not involved in the recruitment and conduct of the study. The information provided by the workers will be kept confidential under scientific numbering and strict management systems. The urine and blood samples provided by the workers will be destroyed after the study. We will disseminate the results to study participants by phone. This study is a cross-sectional survey and not a randomised controlled trials, there is no burden of the intervention.

\section{RESULT}

\section{Characteristics of the coke oven workers}

Table 1 shows the baseline characteristics of the 693 coke plant workers analysed in this study. $\Sigma$ FLU is the sum of urinary 2-OH FLU concentration $(\mathrm{ng} / \mathrm{mL})$ and $3-\mathrm{OH}$ 
Table 1 Sociodemographic and occupational profiles of study group

\begin{tabular}{|c|c|c|c|c|c|c|}
\hline \multirow[b]{2}{*}{ Variable } & \multicolumn{6}{|c|}{ Urinary $\sum$ FLU (ng/mL) } \\
\hline & $\begin{array}{l}\text { Total } \\
(n=693)\end{array}$ & $\begin{array}{l}\text { Q1 (<0.09) } \\
(n=157)\end{array}$ & $\begin{array}{l}\text { Q2 (0.09-0.18) } \\
(n=187)\end{array}$ & $\begin{array}{l}\text { Q3 (0.18-0.34) } \\
(n=174)\end{array}$ & $\begin{array}{l}Q 4(>0.34) \\
(n=175)\end{array}$ & $\mathbf{P}$ \\
\hline \multicolumn{7}{|l|}{ General characteristic } \\
\hline Age (years) & $39.60 \pm 9.72$ & $37.85 \pm 9.01$ & $39.48 \pm 9.82$ & $39.34 \pm 9.82$ & $49.57 \pm 9.86$ & 0.01 \\
\hline Gender (N (\%)) & & & & & & $<0.01$ \\
\hline Male & 465 & $66(42.04)$ & $116(62.03)$ & $135(77.95)$ & $148(84.59)$ & \\
\hline Female & 228 & 91 (57.96) & $71(37.97)$ & 39 (22.05) & $27(15.41)$ & \\
\hline BMI & $20.2 \pm 4.65$ & $24.87 \pm 3.90$ & $24.50 \pm 3.53$ & $25.43 \pm 4.73$ & $25.32 \pm 6.04$ & 0.34 \\
\hline Education (N (\%)) & & & & & & $<0.01$ \\
\hline Junior high school and below & 159 & $24(15.29)$ & $35(18.72)$ & $39(22.41)$ & $61(34.86)$ & \\
\hline Senior high school & 165 & $30(19.11)$ & $48(25.67)$ & $42(24.14)$ & $45(25.71)$ & \\
\hline College and above & 369 & $103(65.61)$ & $104(55.61)$ & $93(53.45)$ & $69(39.43)$ & \\
\hline Smoking status (N (\%)) & & & & & & $<0.01$ \\
\hline Yes & 287 & $27(17.20)$ & $70(37.43)$ & $91(52.30)$ & $99(56.57)$ & \\
\hline No & 406 & $130(82.80)$ & $117(62.57)$ & $83(47.70)$ & $76(43.43)$ & \\
\hline Drinking status (N (\%)) & & & & & & $<0.01$ \\
\hline Yes & 189 & $25(15.92)$ & $57(30.48)$ & $45(25.86)$ & $62(35.43)$ & \\
\hline No & 504 & $132(84.08)$ & $130(69.52)$ & $129(74.86)$ & $113(64.57)$ & \\
\hline $\mathrm{TC}(\mathrm{mmol} / \mathrm{L})$ & $4.95 \pm 0.91$ & $4.79 \pm 0.92$ & $4.90 \pm 0.91$ & $5.06 \pm 0.86$ & $5.04 \pm 0.95$ & 0.02 \\
\hline $\mathrm{TG}(\mathrm{mmol} / \mathrm{L})$ & $1.47 \pm 1.14$ & $1.29 \pm 0.72$ & $1.28 \pm 0.70$ & $1.68 \pm 1.51$ & $1.64 \pm 1.35$ & $<0.01$ \\
\hline $\mathrm{HDL}$ (mmol/L) & $1.25 \pm 0.18$ & $1.28 \pm 0.18$ & $1.24 \pm 0.17$ & $1.23 \pm 0.19$ & $1.27 \pm 0.19$ & 0.04 \\
\hline $\mathrm{LDL}$ (mmol/L) & $2.96 \pm 0.88$ & $2.89 \pm 0.88$ & $2.90 \pm 0.88$ & $3.06 \pm 0.87$ & $3.00 \pm 0.88$ & 0.20 \\
\hline Plasma viscosity (mPa.s,30/s) & $4.60 \pm 2.67$ & $4.00 \pm 2.50$ & $4.47 \pm 2.68$ & $4.36 \pm 2.84$ & $5.52 \pm 2.42$ & $<0.01$ \\
\hline \multicolumn{7}{|l|}{ PAHs internal exposure biomarker } \\
\hline 2-OH NAP (ng/mL) & $0.64 \pm 1.57$ & $0.55 \pm 1.41$ & $0.63 \pm 2.25$ & $0.65 \pm 1.19$ & $0.73 \pm 1.09$ & 0.76 \\
\hline 1-OH NAP (ng/mL) & $0.06 \pm 0.24$ & $0.03 \pm 0.04$ & $0.04 \pm 0.06$ & $0.05 \pm 0.09$ & $0.12 \pm 0.45$ & $<0.01$ \\
\hline 1-OH PYR (ng/mL) & $0.20 \pm 0.50$ & $0.07 \pm 0.05$ & $0.10 \pm 0.08$ & $0.14 \pm 0.11$ & $0.48 \pm 0.93$ & $<0.01$ \\
\hline
\end{tabular}

BMI, Body Mass Index; FLU, fluorine; NAP, naphthalene; PYR, pyrene.

FLU concentration $(\mathrm{ng} / \mathrm{mL})$. We put the population into a quartile based on $\Sigma$ FLU concentrations in table 1 . It is shown that people exposed to high concentrations of $\Sigma$ FLU are older men who are smoking and drinking. The blood lipid level of the high exposure group is also higher than the low exposure group. There was also a statistically significant difference in dietary habits and monthly income differences between these groups. Except for OH-NAP1 and OH-NAP2, other OH-PAHs of the high exposure group are higher. Plasma viscosity was also higher in the high exposure group, and the difference was statistically significant.

\section{General linear model of OH-PAHs and plasma viscosity}

We used two different models to test the relationship between concentration of OH-PAHs in urine and plasma viscosity and present the results in table 2 . In generalised linear model, plasma viscosity was positively associated with 2-OH FLU, 3-OH FLU and $\Sigma$ FLU. (Adjustment for age, gender, smoking, drinking, BMI, education, income, fruit, grease, TG, TC, HDL, LDL.)

\section{Stratified analysis of OH-FLU2, OH-FLU3, $\Sigma$ FLU and plasma} viscosity

Then, the subjects were divided into four groups with the quartile of 2-OH FLU concentrations, 3-OH FLU concentrations and $\Sigma$ FLU concentrations. After adjustment for age, gender, smoking, drinking, BMI, education, income, fruit, grease, TG, TC, HDL, LDL, and made the result into figure 1. We found that concentration of 2-OH FLU and $\Sigma$ FLU has a dose-response relationship with plasma viscosity at a high level and the trend test is statistically significant ( $\mathrm{p}$ trend $=0.02,0.01$ ). The adjusted $\beta$ for higher plasma viscosity risk in the highest quartile was $0.70(0.13-1.27)$ for 2-OH FLU, 0.97 (0.39-1.55) for $\Sigma$ FLU, respectively.

Cubic limit spline graph of OH-PAHs and plasma viscosity Then we made a cubic limit spline graph of OH-PAHs and plasma viscosity made it to figure 2 . The results from 
Table 2 Associations between PAH metabolites and plasma viscosity by multiple linear regression in different models

\begin{tabular}{|c|c|c|}
\hline & \multicolumn{2}{|l|}{$\beta(95 \% \mathrm{Cl})$} \\
\hline & Model 1 & Model 2 \\
\hline 2-OH NAP & $-0.03(-0.15$ to 0.10$)$ & $-0.03(-0.15$ to 0.09$)$ \\
\hline 1-OH NAP & $-0.54(-1.37$ to 0.30$)$ & $-0.51(-1.30$ to 0.27$)$ \\
\hline 3-OH FLU & 10.46 (6.73 to 14.20$)$ & 7.12 (3.39 to 10.85$)$ \\
\hline 2-OH FLU & 1.62 (1.02 to 2.22$)$ & 1.14 (0.54 to 1.74$)$ \\
\hline 2-OH PHE & 1.27 (0.65 to 1.89$)$ & 0.88 (0.29 to 1.48$)$ \\
\hline 9-OH PHE & 0.76 (0.14 to 1.38$)$ & $0.43(-0.17$ to 1.04$)$ \\
\hline 1-OH PHE & 3.95 (2.07 to 5.82 ) & 2.81 (1.01 to 4.61 ) \\
\hline 1-OH PYR & 0.75 (0.35 to 1.14$)$ & 0.49 (0.11 to 0.87$)$ \\
\hline$\Sigma$ FLU & 1.52 (0.98 to 2.05$)$ & $1.07(0.53$ to 1.61$)$ \\
\hline$\Sigma$ NAP & $-0.03(-0.16$ to 0.09$)$ & $-0.04(-0.15$ to 0.08$)$ \\
\hline$\Sigma$ PHE & 0.58 (0.28 to 0.88$)$ & 0.38 (0.09 to 0.67$)$ \\
\hline
\end{tabular}

Model 1 unadjusted.

Model 2 adjusted for age, gender, smoking, drinking, BMI, education, income, fruit, grease, TG, TC, HDL, LDL.

FLU, fluorine; HDL, high-density lipoprotein; LDL, low-density lipoprotein; NAP, naphthalene; PAH, polycyclic aromatic hydrocarbon; PYR, pyrene; TG, Triglyceride.

restricted cubic spline models (adjusted by age, gender, smoking, drinking, BMI, education, income, fruit, grease, TG, TC, HDL, LDL) show that urinary 2-OH FLU, 3-OH

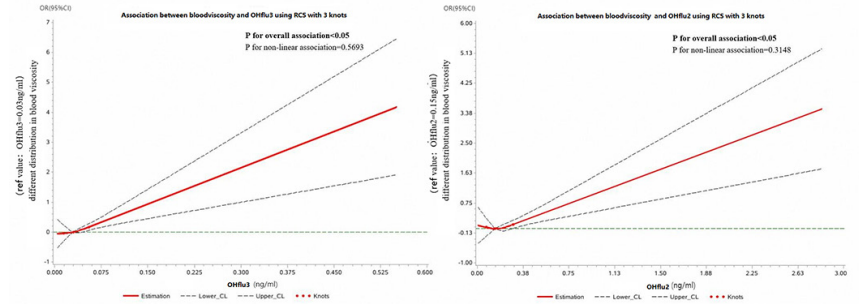

Figure 2 Cubic limit spline graph of $\mathrm{OH}-\mathrm{FLUs}$ and plasma viscosity. Adjusted for age, gender, smoking, drinking, BMI, education, income, fruit, grease, TG, TC, HDL, LDL. FLU, fluorine.

FLU, concentrations $(\mathrm{ng} / \mathrm{mL})$ have a linear association with plasma viscosity. ( $\mathrm{P}$ for overall association $<0.05$, and $\mathrm{P}$ for non-linear association $>0.05$.) The correlation between other metabolites in urine and plasma viscosity has no linear statistical significance.

\section{Sensitivity analysis}

We put other OH-PAHs into a linear model for sensitivity analysis (includes three OH-PAHS with low detection rates) and present the results in table 3 . It was found that when the adjusted variables in the model did not include OH-FLU2 or OH-FLU3, the relationship between PAH metabolites of fluorene PAH metabolites of fluorene and plasma viscosity was still positively correlated and statistically significant. However, the relationship between OH-FLU3 and plasma viscosity was not statistically

Figure 1 Stratified analysis of $\mathrm{OH}-\mathrm{FLU} 2, \mathrm{OH}-\mathrm{FLU} 3, \Sigma \mathrm{FLU}$ and plasma viscosity. Adjusted for age, gender, smoking, drinking, BMI, education, income, fruit, grease, TG, TC, HDL, LDL. FLU, fluorine. 


\begin{tabular}{llll} 
Table 3 & Sensitivity analysis & & \\
\hline & $\boldsymbol{\beta}$ & $\mathbf{9 5 \%} \mathbf{C l}$ & $\mathbf{P}$ \\
\hline OHFLU2 * $^{*}$ & 1.13 & 0.23 to 2.03 & 0.01 \\
\hline OHFLU3 * $^{*}$ & 5.99 & 1.47 to 10.50 & 0.01 \\
\hline FLU * & 1.10 & 0.29 to 1.91 & 0.01 \\
\hline OHFLU2† & 0.70 & -0.35 to 1.75 & 0.19 \\
OHFLU3 & 4.18 & -1.07 to 9.44 & 0.12 \\
\hline
\end{tabular}

*Adjusted for age, gender, smoking, drinking, BMI, education, income, fruit, grease, TG, TC, HDL, LDL, OHNAP1, OHNAP2, OHPHE1, OHPHE2, OHPHE9, OHPYR1, OHCHR3, OHCHR6, OHBAP9.

†Adjusted for a and OHFLU3.

$\ddagger$ Adjusted for a and OHFLU2.

FLU, fluorine; NAP, naphthalene; PHE, phenanthrene; PYR, pyrene.

significant after adding OH-FLU2 as an adjustment variable to the linear model $(\mathrm{p}=0.12)$. OH-FLU2 is the same $(\mathrm{p}=0.19)$.

\section{DISCUSSION}

We demonstrated for the first time that PAHs had an adverse effect on plasma viscosity. The results showed a significant association of 2-OH FLU level with high plasma viscosity. Plasma viscosity increased by $1.62(\mathrm{mPa} . \mathrm{s}, 30 / \mathrm{s})$ for each ng/L of 2-OH FLU after adjusting for age, sex, smoking, drinking, BMI, education, income, fruit, grease, TG, TC, HDL and LDL. Plasma viscosity increased by 1.13 (mPa.s,30/s) for each $\mathrm{ng} / \mathrm{mL}$ of 2-OH FLU. The effects of PAHs on CVD have been widely investigated, and the alterations in plasma viscosity could cause haemodynamic disturbances that may promote the formation of atherosclerosis. ${ }^{15}$ Our study demonstrates that plasma viscosity would change with the urinary levels of $2-\mathrm{OH}$ FLU in healthy coke oven workers. The results of our study provide a new marker for coke oven workers who are exposed to PAHs to prevent CDV.

People are exposed to PAHs in daily life. The level of $\mathrm{OH}-\mathrm{PAHs}$ in urine is widely used as a biomarker of PAH exposure. $^{16}{ }^{17}$ In our study, per cent detection of 2-OH NAP, 1-OH Nap, 3-OH FLU, 2-OH FLU, 9-OH PHE, 2-OH PHE, 1-OH PHE and 1-OH PYR was higher. However, the per cent detection of 3-OH CHR, 6-OH CHR and 9-OH Bap was minimal. This is likely because chrysene has a $4-5$ ring structure, and it is more likely to be eliminated from the faeces, so the concentration of metabolites in urine is lower. ${ }^{13}{ }^{18}$ In addition, $1.2 \%-13.4 \%$ of the exposure of PAHs in coke oven gas is pyrene, which mainly converts to $1-\mathrm{OH}$ PYR after entering the human body, 1-OH PYR is the most commonly used OH-PAH biomarker. ${ }^{19}$ In our study, the GMSG $( \pm \mathrm{GSD})$ of urinary 1-OHPYR detected in coke oven workers of $0.2 \pm 0.5 \mathrm{ng} / \mathrm{mL}$ is similar to the urban site's participants (MANHYIA) in Bortey-Sam et als study, which GMSG ( \pm GSD) of urinary 1-OHPYR is $0.208 \pm 4.16 \mathrm{ng} / \mathrm{mL} .{ }^{20}$

The plasma viscosity of all coke oven workers is 5.57 (mPa.s,30/s) in our study, and the mean \pm SD of plasma viscosity is $4.60 \pm 2.67$ (mPa.s,30/s). According to the medical reference value (4.43-9.45), the plasma viscosity values of most workers were within the medical reference range. We found some papers working on the relationship between haemorheological changes and PAH concentration to find out if there are serum biomarkers of CVD, which is related to PAHs. Abou-Elwafa et al observed that the mean haemoglobin level and RBC count of healthy male service and office workers were significantly higher than those of the petrol station attendants. ${ }^{21}$ Another study focused on the relationship between PAH exposure and serum biomarkers of $\mathrm{CVD},{ }^{22}$ though these studies failed. However, past studies have found that people exposed to high levels of PAHs might have increased levels of white blood cells, CRP and fibrinogen. ${ }^{23}{ }^{24}$ In our study, we can also see that there is an association between OH-PAH and plasma viscosity after reaching a certain dose (2-OH FLU $>0.29 \mathrm{ng} / \mathrm{mL}$ CI 0.13 to 1.27). Therefore, this kind of association may be achieved with a certain dose, which makes sense for CVD prevention of coke oven workers.

Fluorene-related biological mechanisms of plasma viscosity are unclear. Studies about fluorene are rare, so we checked the studies working on the relationship between PAHs and CVD. After checking the studies on both humans and animals, we found a possible mechanism. One study has shown that coke oven workers exposed to PAHs may cause an acute systemic reaction increase and lead to a higher risk of CVD ${ }^{25}$ and CRP, a serum biomarker of inflammation has been shown to be correlated with high concentrations of OH-PAHs in multiple studies, ${ }^{2627}$ and CRP is one of the main factors affecting plasma viscosity. The change in plasma viscosity may be due to the inflammatory effect.

PAHs are associated with multiple CVD, like ASCVD, hypertension, etc. Studies have shown that many inflammatory factors are possible promoters of atherosclerotic plaques. ${ }^{28}$ The number of inflammatory cells in atherosclerotic plaques significantly increased in the case of chronic PAH exposure in an animal experiment. ${ }^{29}$ The relationship between PAHs and hypertension has been discovered in several studies. One study has shown that PAHs in housewives' hair is associated with hypertension, ${ }^{30}$ and PAHs have been found to be associated with prehypertension in a study of children in Saudi Arabia. ${ }^{31}$ PAHs are associated with many chronic CVDs. Therefore, it may be more meaningful to find that plasma viscosity increase in the normal plasma viscosity range in healthy workers, indicating that plasma viscosity is related to chronic CVDs caused by PAHs. It is very sensitive in the early stage of the disease and is of higher value for prevention.

Our research has the following limitations. First, the population we studied was not a population already suffering from CVD or ASCVD, and it was not certain that changes in plasma viscosity were related to risk of CVD or ASCVD. Second, plasma fibrinogen concentration is a major factor affecting plasma viscosity. But our database does not have fibrinogen data. Therefore, we are not sure whether the change in plasma viscosity is mainly due to CVD. Third, the lack of a control group, a complete occupational history and 
a complete clinical history. Finally, although we considered several important confounders in the analysis, our study does not include other confounding factors not measured or selected, such as genetic factors.

\section{CONCLUSION}

In conclusion, the results of this study could provide evidence for a relationship between PAH exposure (as measured by urinary levels of PAH metabolites) and biomarker of CVD (plasma viscosity).

Acknowledgements This study has been supported by the National Natural Science Foundation of China (81673143, 81072279 and 30800899), the Shanxi Province Natural Science Foundation of China (2015011128, 2010021034-3), the Shanxi Scholarship Council of China (2016-057). The authors thank the participants and medical staff, who assisted with the sample collection and neurobehavioral assessment and workers who provide blood and urine samples.

Contributors MW: writing — original draft, methodology, formal analysis, writingreview and editing. CL: investigation, data curation, resources, writing - review and editing. HW: investigation, writing — review and editing. JN: funding acquisition, conceptualisation, supervision, project administration, writing — review and editing. JY: writing —review and editing. JN is the guarantor of this article.

Funding This study has been supported by the National Natural Science Foundation of China (81673143).

Competing interests None declared.

Patient consent for publication Not applicable.

Ethics approval The authors declare that this study has been approved by the Ethics Committee of Shanxi Medical University. The serial number is 2019LL236.

Provenance and peer review Not commissioned; externally peer reviewed.

Data availability statement Data are available upon reasonable request. No additional data available.

Supplemental material This content has been supplied by the author(s). It has not been vetted by BMJ Publishing Group Limited (BMJ) and may not have been peer-reviewed. Any opinions or recommendations discussed are solely those of the author(s) and are not endorsed by BMJ. BMJ disclaims all liability and responsibility arising from any reliance placed on the content. Where the content includes any translated material, BMJ does not warrant the accuracy and reliability of the translations (including but not limited to local regulations, clinical guidelines, terminology, drug names and drug dosages), and is not responsible for any error and/or omissions arising from translation and adaptation or otherwise.

Open access This is an open access article distributed in accordance with the Creative Commons Attribution Non Commercial (CC BY-NC 4.0) license, which permits others to distribute, remix, adapt, build upon this work non-commercially, and license their derivative works on different terms, provided the original work is properly cited, appropriate credit is given, any changes made indicated, and the use is non-commercial. See: http://creativecommons.org/licenses/by-nc/4.0/.

\section{ORCID iD}

Jisheng Nie http://orcid.org/0000-0002-8194-3101

\section{REFERENCES}

1 Gao P, SE D, Hou L. Human exposure to polycyclic aromatic hydrocarbons. Metabolomics perspective 2018;119:466.

$2 \mathrm{Kim} \mathrm{K-H,} \mathrm{Jahan} \mathrm{SA,} \mathrm{Kabir} \mathrm{E,} \mathrm{et} \mathrm{al.} \mathrm{A} \mathrm{review} \mathrm{of} \mathrm{airborne} \mathrm{polycyclic}$ aromatic hydrocarbons (PAHs) and their human health effects. Environ Int 2013;60:71-80.

$3 \mathrm{Xu}$ X, Cook RL, llacqua VA, et al. Studying associations between urinary metabolites of polycyclic aromatic hydrocarbons (PAHs) and cardiovascular diseases in the United States. Sci Total Environ 2010;408:4943-8.

$4 \mathrm{Hu} \mathrm{C}$, Hou J, Zhou Y. Association of polycyclic aromatic hydrocarbons exposure with atherosclerotic cardiovascular disease risk: a role of mean platelet volume or Club cell secretory protein 2017;233:45-53.
5 Shah ASV, Lee KK, McAllister DA, et al. Short term exposure to air pollution and stroke: systematic review and meta-analysis. BMJ 2015;350:h1295.

6 Lee KK, Miller MR, Shah ASV. Air pollution and stroke. J Stroke 2018;20:2-11.

7 Toth K, Habon T, Horvath I, et al. Hemorheological and hemodynamical parameters in patients with ischemic heart disease at rest and at peak exercise. Clin Hemorheol Microcirc 1994;14:329-38.

8 Kesmarky G, Toth K, Habon L. Hemorheological parameters in coronary artery disease 1998;18:245-51.

9 Lowe GDOJBCH. Blood rheology in vitro and in vivo 1987:1:597-636.

10 Danesh J, Collins R, Peto R, et al. Haematocrit, viscosity, erythrocyte sedimentation rate: meta-analyses of prospective studies of coronary heart disease. Eur Heart J 2000;21:515-20.

11 Lowe GD, Lee AJ, Rumley A. Blood viscosity and risk of cardiovascular events: the Edinburgh artery study 2015;96:168-73.

12 John CD, Berrin S, David LJ. Exposure to polycyclic aromatic hydrocarbons and serum inflammatory markers of cardiovascular disease 2012;117:132-7.

13 Zhao G, Chen Y, Wang S, et al. Simultaneous determination of 11 monohydroxylated PAHs in human urine by stir bar sorptive extraction and liquid chromatography/tandem mass spectrometry. Talanta 2013;116:822-6.

$14 \mathrm{Nie}$ J, Li J, Cheng L, et al. Maternal urinary 2-hydroxynaphthalene and birth outcomes in Taiyuan, China. Environ Health 2018;17:91.

15 Baskurt, Oguz K. Handbook of hemorheology and hemodynamics. IOS Press, 2007.

16 Ying G, Kurunthachalam S, Husam A. Concentrations and profiles of urinary polycyclic aromatic hydrocarbon metabolites (OH-PAHs) in several Asian countries 2013;47:2932-8.

17 Gong J, Zhu T, Kipen H, et al. Urinary polycyclic aromatic hydrocarbon metabolites as biomarkers of exposure to trafficemitted pollutants. Environ Int 2015;85:104-10.

18 Zhou Y, Hu X-xue, Li T, et al. Similarity type of general solution for one-dimensional heat conduction in the cylindrical coordinate. Int $J$ Heat Mass Transf 2018;119:542-50.

19 Srogi K. Monitoring of environmental exposure to polycyclic aromatic hydrocarbons: a review. Environ Chem Lett 2007;5:169-95.

20 Bortey-Sam N, Ikenaka Y, Akoto O, et al. Oxidative stress and respiratory symptoms due to human exposure to polycyclic aromatic hydrocarbons (PAHs) in Kumasi, Ghana. Environ Pollut 2017;228:311-20.

21 Abou-EIWafa HS, Albadry AA, El-Gilany A-H, et al. Some biochemical and hematological parameters among petrol station attendants: a comparative study. Biomed Res Int 2015;2015:1-6.

22 Clark JD, Serdar B, Lee DJ, et al. Exposure to polycyclic aromatic hydrocarbons and serum inflammatory markers of cardiovascular disease. Environ Res 2012;117:132-7.

23 Rückerl R, Ibald-Mulli A, Koenig W, et al. Air pollution and markers of inflammation and coagulation in patients with coronary heart disease. Am J Respir Crit Care Med 2006;173:432-41.

24 Rückerl R, Greven S, Ljungman P, et al. Air pollution and inflammation (interleukin-6, C-reactive protein, fibrinogen) in myocardial infarction survivors. Environ Health Perspect 2007;115:1072-80.

25 Hadrup N, Mielżyńska-Švach D, Kozłowska A, et al. Association between a urinary biomarker for exposure to PAH and blood level of the acute phase protein serum amyloid $A$ in coke oven workers. Environ Health 2019;18:81.

26 Everett CJ, King DE, Player MS, et al. Association of urinary polycyclic aromatic hydrocarbons and serum C-reactive protein. Environ Res 2010;110:79-82.

27 Alshaarawy O, Zhu M, Ducatman A, et al. Polycyclic aromatic hydrocarbon biomarkers and serum markers of inflammation. A positive association that is more evident in men. Environ Res 2013;126:98-104.

28 Myers GL, Rifai N, Tracy RP, et al. CDC/AHA workshop on markers of inflammation and cardiovascular disease. Circulation 2004;110:543-4.

29 Polycyclic aromatic hydrocarbons induce an inflammatory atherosclerotic plaque phenotype irrespective of their DNA binding properties 2005;19:1290-2.

30 Wang B, Li Z, Ma Y, et al. Association of polycyclic aromatic hydrocarbons in housewives' hair with hypertension. Chemosphere 2016;153:315-21.

31 Trasande L, Urbina EM, Khoder M, et al. Polycyclic aromatic hydrocarbons, brachial artery distensibility and blood pressure among children residing near an oil refinery. Environ Res 2015;136:133-40. 